\title{
El outsourcing en el desempeño de las exportaciones de las empresas
}

Sergio Garcilazo

Lagunes

Universidad Panamericana sgarcila@up.edu.mx

\section{Resumen}

En este artículo se examina el efecto del outsourcing en el desempeño de las exportaciones de las empresas. El análisis se basa en las teorías de los negocios internacionales y el punto de vista de los recursos y la economía de los costos de transacción, según los cuales el outsourcing en el extranjero ayuda a las empresas — directa o indirectamente - a exportar más, a reducir sus costos de producción y a aumentar la flexibilidad de la empresa; además, puede proporcionar nuevos recursos y conocimientos del mercado. Sin embargo, el impacto del outsourcing internacional depende de los recursos y las capacidades de las empresas para gestionar una red de proveedores extranjeros y para absorber los conocimientos extranjeros. Usando una base de datos de alrededor de mil empresas de fabricación en México de 2007, se encontró que las operaciones de outsourcing en el extranjero aumenta el rendimiento de la exportación, los efectos son más fuertes en los mercados de exportación de donde las empresas importan los bienes intermedios. Los resultados también muestran que el tamaño de la empresa, la organización de las importaciones intrafirma y la experiencia en la exportación moderan los efectos del outsourcing de manera positiva. Estos resultados tienen implicaciones para las empresas y los políticos responsables del diseño de políticas.

Palabras claves: outsourcing, exportaciones, empresas multinacionales. 


\title{
The outsourcing in the export performance of the firms
}

\begin{abstract}
This paper examines the effect of outsourcing on the export performance of firms. The analysis is based on the theories of international business and the point of view of the resources and the economics of transaction costs, according to which offshore outsourcing helps companies - directly or indirectly - to enhanced exports, production costs reduction and to increase the firm flexibility; also providing new resources and marketing knowledge. Nevertheless, the outsourcing effects depend upon the resources and capabilities that the firms have to manage a foreign providers network and to absorb the foreign knowledge. Using a database of about 1,000 manufacturing companies in Mexico in 2007, it was found that offshore outsourcing increases the performance of exports; the effects are stronger in export markets where firms import intermediate goods. The results also show that the size of the company, the organization of intrafirm imports and export experience moderate the effects of outsourcing in a positive way. These results have implications for businesses and policy makers.
\end{abstract}

Keywords: outsourcing, exports, multinational corporations.

\section{Introducción}

En los últimos años, el outsourcing ha llamado la atención de los medios de comunicación, políticos y académicos (Rasheed y Gilley, 2005). También se observa un rápido crecimiento de ésta, gracias a la liberalización del comercio y al progreso tecnológico; asimismo, se piensa que crecerá en los sectores manufactureros y de servicios, lo cual generará preocupación en muchos países desarrollados. Cabe aclarar que en los países desarrollados el outsourcing externo de los países en desarrollo, en particular, es a menudo acusado de la disminución de empleo; sin embargo, podría aportar beneficios importantes para los países desarrollados. Además de los ingresos y ahorro de costos, Farrell (2005) sostiene que la externalización al extranjero ayuda a los países desarrollados a aumentar sus ventas de exportación y, por lo tanto, el empleo, pues ésta estimula el crecimiento macroeconómico de los países en desarrollo y, en consecuencia, aumenta sus importaciones.

Según lo propuesto por Friedman (2004), "lo que pasa alrededor viene alrededor". El outsourcing no es obviamente un desafío único para los países desarrollados. En los mercados globales la externalización al extranjero es reconocida con frecuen- 
cia como una estrategia esencial de las empresas para mantener y desarrollar sus ventajas competitivas (Kotabe y Mudambi, 2009).

Motivados por este problema nuestro objetivo es ir más allá, tanto en la evidencia anecdótica como en el punto de vista macroeconómico para las industrias de fabricación, pues se investiga empíricamente los efectos microeconómicos del outsourcing en los resultados de exportación. Para ello, se toman como base las teorías del comercio internacional, el punto de vista de los recursos y la economía de los costos de transacción, que argumentan que la externalización al extranjero aumenta — directa e indirectamente - la capacidad de las empresas para exportar. Esto se hace mediante la reducción de sus costos de producción, aumentando su flexibilidad y dando nuevos recursos y conocimiento del mercado. Sin embargo, podría ser arriesgado y costoso para aquellas empresas dedicadas al outsourcing en el extranjero si no toman en cuenta los diferentes problemas operativos y estratégicos.

Por otra parte, se puede señalar que las diferencias en los recursos y capacidades de las empresas para administrar una red de proveedores extranjeros, así como para absorber los conocimientos extranjeros, podrían conducir a la variación en los efectos del outsourcing entre empresas. Para llevar a cabo esto, se utilizó una base de datos única de alrededor de mil empresas y las filiales de propiedad extranjera establecidas en México en 2007. Los datos abarcan las empresas multinacionales pequeñas, medianas y grandes en la industria manufacturera, las cuales representan alrededor del $70 \%$ de las importaciones y las exportaciones de manufactura mexicana. Además, se define la externalización al extranjero de bienes intermedios suministrados por proveedores extranjeros independientes (Kotabe y Murray, 2004; Mol, Van Tulder, y Beije, 2005).

Encontramos que, ceteris paribus, el outsourcing mejora el desempeño de las exportaciones de las empresas multinacionales; sin embargo, el impacto es más fuerte cuando las importaciones de bienes intermedios son desde el país al que se realizan las exportaciones. El impacto es moderado positivamente por el tamaño de la empresa, la organización de las importaciones intrafirma y la experiencia de la exportación, lo cual nos confirma que el outsourcing en las empresas multinacionales podría dar ventajas competitivas globales y, adicionalmente, que la extensión de los beneficios puede diferirse a través de diferentes empresas; por último, se sugiere que las medidas proteccionistas en contra de la externalización al extranjero podrían reducir la competitividad internacional de las industrias nacionales $\mathrm{y}$, por lo tanto, ser contraproducente. 
Carecemos de evidencia empírica microeconómica en el outsourcing externo; aunque existen estudios más empíricos para examinar sus determinantes (Doh, Bunyaratavej y Hahn, 2009), hasta donde sabemos sólo Mol et al. (2005) y Di Gregorio, Musteen, y Thomas (2009) han investigado el impacto del outsourcing. De una muestra de doscientas empresas holandesas, Mol et al. (2005) no encontraron efectos significativos en los resultados económicos y financieros. En un estudio de mayor contribución, y utilizando una muestra de cien empresas de EE.UU., Di Gregorio et al. (2009) concluyen que la externalización al extranjero aumenta el rendimiento de las exportaciones de las pequeñas y medianas empresas (Pyme). Es por ello que la contribución de este trabajo es complementario a la literatura de administración.

Empíricamente, Di Gregorio et al. (2009) estudiaron a las pequeñas y medianas empresas ${ }^{1}$ multinacionales; además, hizo hincapié en el outsourcing de servicios administrativos y técnicos. Se centró en las empresas multinacionales de diferentes tamaños y en el examen del outsourcing de fabricación utilizando datos primarios sobre una pequeña muestra de las empresas; en cambio, nuestros datos secundarios incluyen un gran número de empresas e información en los niveles de la empresa y el país.

También hay que señalar que nuestras predicciones teóricas son diferentes. Los autores señalados sugieren - pero no la prueban- que el outsourcing no puede ser beneficioso para las grandes empresas multinacionales (EMN). Se argumenta que las empresas multinacionales tienen más probabilidades de ganar de la externalización al extranjero a causa de una mayor capacidad de absorción de recursos de producción y los recursos para coordinar la dispersión geográfica de la red de los proveedores (Levy, 2005); sin embargo, se tiene en cuenta la existencia de la heterogeneidad entre las empresas multinacionales.

\section{Antecedentes}

Las teorías de los negocios internacionales identifican tres canales principales a través de los cuales el outsourcing podría aumentar las ventajas competitivas de las empresas y con ello indirectamente sus ventas de exportación (Kotabe y Murray, 2004; Kotabe y Mudambi, 2009).

${ }^{1}$ Su muestra contiene sólo el 13\% de las empresas multinacionales, pequeñas y medianas empresas. 
En primer lugar, el outsourcing internacional puede permitir a las empresas reducir sus costos de producción. Las empresas de outsourcing podrían comprar insumos más baratos de los proveedores extranjeros (Rasheed y Gilley, 2005), quienes pueden pagar salarios más bajos en los países en desarrollo; mientras que en los países desarrollados pueden disfrutar de una tecnología superior o beneficiarse de economías de escala más grande. En segundo lugar, el outsourcing permite una mayor flexibilidad porque la empresa de este tipo está menos comprometida con un tipo específico de tecnología; además, permite a las empresas reasignar sus recursos en aquellas actividades que son sus competencias básicas. En tercer lugar, la externalización al extranjero ayuda a las empresas a acceder a los recursos y al conocimiento de los proveedores extranjeros (Grant, 1991), lo cual les permite complementar su inventario de recursos y aprovechar los nuevos conocimientos que, de otro modo, se pueden acceder en la empresa y/o en el país de origen. En general, los investigadores ven al comercio internacional como un importante canal de difusión no sólo de conocimientos tecnológicos y de organización, sino también de información de mercado (Karlsen, Silseth, Benito, y Welch, 2003; Salomon y Jin, 2008) cuando en la búsqueda y negociación con proveedores extranjeros las empresas desarrollan su red de contactos en el extranjero y aprenden acerca de estos mercados.

Esto a su vez puede aumentar la transferencia de los recursos que las empresas utilizan para exportar. Por ejemplo, mientras que la tecnología es vista como altamente fungible, los recursos de mercadotecnia no (Salomon y Shaver, 2005). En resumen, el outsourcing podría proporcionar a las empresas ventajas de exportación indirecta a través de la reducción de costos, mayor flexibilidad, obtención de recursos adicionales y/o conocimiento del mercado. Por lo tanto:

Hipótesis 1: El outsourcing internacional se relaciona positivamente con los resultados de exportación.

En relación con esta hipótesis, se argumenta que los beneficios del outsourcing internacional podrían ser más directos y, en consecuencia, más fuertes cuando los mercados de importación y exportación son idénticos, como en la anécdota de Friedman acerca de las importaciones y las exportaciones de EE.UU. a la India.

El valor de los conocimientos relacionados con el mercado del outsourcing es mayor cuando las empresas de exportación se enfocan al país importado; a través de interacciones con los proveedores y otros agentes en el país importador, las 
empresas pueden aprender las preferencias del consumidor sobre los productos específicos y los productos competidores (Karlsen et al., 2003; Welch y Luostarinen, 1993.); asimismo, estas empresas se familiarizan con el medio ambiente local único, con la reducción de las desventajas específicas de cada lugar (Barkema y Pennings, 1996) y con los costos de transacción específica a la exportación (Verwaal y Donkers, 2002). Por otra parte, las ventajas de los proveedores son, por definición, más locales (Anand y Delios, 1997), por lo que para las empresas exportadoras no es necesario adaptar los insumos importados a la situación del mercado de exportación (a las normas técnicas diferentes) o para incurrir en costos para la personalización; ${ }^{2}$ además, los proveedores son más sensibles a los cambios en su mercado. La importación de insumos del mercado de exportación podría ser una condición esencial para la exportación, como en el caso del countertrade ${ }^{3}$ (Choi, Lee y Kim, 1999) que a pesar de su importancia es difícil de establecer. Sin embargo, los gobiernos podrían obligar a las empresas a que incorporen insumos elaborados en el país de exportación en sus productos finales. El countertrade también puede ser adoptado voluntariamente para entrar en mercados caracterizados por fuertes limitaciones ambientales y las imperfecciones del mercado. En resumen, el outsourcing en el país de exportación puede dar a las empresas los recursos específicos de cada país y el conocimiento. Por lo tanto:

Hipótesis 2: Ceteris paribus, los efectos positivos del outsourcing en los resultados de exportación son más altos en los mercados de exportación, de donde las empresas importan los bienes intermedios.

Anteriormente se explicó por qué las empresas deciden el outsourcing en el extranjero con el fin de incrementar sus ventajas competitivas, y cómo el outsourcing, en consecuencia, podría contribuir a los resultados de exportación, aunque aquél podría ser costoso y arriesgado (Mesquita, Anand, y Brush, 2008). Ahora bien, sus costos incluyen no sólo los costos de adquisición, sino también los de transacción, ex ante (búsqueda y negociación de los costos) y ex post (costos de supervisión y ejecución), como se destaca en la literatura de las expresiones culturales tradicionales (Leiblein, Reuer, y Dalsace, 2002).

\footnotetext{
${ }^{2}$ Un producto final podría estar compuesto por un componente común producido en el país y un componente de productos específicos adaptados a cada país de acogida (véase, por ejemplo, la literatura en común).

${ }^{3}$ Intercambio compensado, forma de hacer comercio internacional.
} 
Las empresas de outsourcing podrían ser más dependientes de sus proveedores y perder el control de la actividad que se externaliza; además, podrían ser objeto de un comportamiento oportunista y no hay que olvidar que el outsourcing internacional incurre en costos de coordinación. Los costos de transacción y la coordinación son propensos a ser mayores en el caso de la externalización en el extranjero, debido a la "responsabilidad del extranjero" y a la distancia geográfica con los proveedores extranjeros. Estos costos podrían ser excesivos cuando las empresas no tienen suficientes recursos tecnológicos y de organización o la capacidad para supervisar y coordinar sus relaciones con los proveedores remotos. Por lo que las empresas también necesitan una capacidad de absorción para asimilar, combinar y utilizar los recursos externos y el conocimiento (Zahra y George, 2002).

En este contexto, se indica que las empresas multinacionales poseen en promedio ventajas decisivas frente a las empresas no multinacionales en el outsourcing internacional. ${ }^{4}$ Estas ventajas críticas pueden explicar por qué el efecto neto de la externalización al extranjero podría ser positivo para las empresas multinacionales, las cuales tienen mayores recursos y reducen la transacción y los costos de coordinación del outsourcing. Por otra parte, su capacidad de absorción también es probable que sea mayor; al respecto Rangan (2000) explica que las empresas multinacionales enfrentan menores costos de búsqueda (es decir, la identificación de los socios de cambio) y la deliberación (la evaluación de su capacidad y fiabilidad). Al operar en diferentes países, las empresas multinacionales están incrustadas en las redes sociales bien elaboradas y tienen amplias relaciones de negocios. Levy (2005) hace hincapié en que las empresas multinacionales, con el tiempo, han aumentado su capacidad organizativa y tecnológica para dividir y coordinar la dispersión geográfica de la red de proveedores.

Sin embargo, los recursos podrían diferir no sólo entre las empresas multinacionales y las empresas no multinacionales, sino también entre las empresas multinacionales, lo que lleva a algunas diferencias en los efectos netos del outsourcing en las empresas multinacionales. Si bien las empresas multinacionales parecen ser más grandes en promedio que las empresas no multinacionales, no todas las empresas multinacionales son las grandes corporaciones (Navaretti y Venables, 2004). Las empresas multinacionales también incluyen las Pyme $\mathrm{P}^{5}$ argumentan

\footnotetext{
${ }^{4}$ UNCTAD define una empresa multinacional como una empresa que controla los activos de por lo menos una entidad en el extranjero.

${ }^{5}$ Básicamente, la distinción entre las Pyme y las grandes empresas se basa en el nivel de empleo.
} 
que las empresas multinacionales pueden diferir en su tamaño y, por lo tanto, en sus recursos financieros y de gestión (Dhanaraj y Beamish, 2003) 6 . Por lo tanto, las empresas multinacionales más grandes podrían beneficiarse en mayor porcentaje por el outsourcing, pues poseen un mayor stock de los recursos administrativos y financieros, así como de las capacidades para gestionar su cadena de suministro.

Las grandes empresas multinacionales cuentan con la experiencia y mano de obra para crear sofisticados sistemas, contratación basada en la tecnología de apoyo (Elmaghraby, 2000) y para reducir los costos de coordinación con los proveedores extranjeros. Asimismo, estas empresas pueden obtener economías de escala en la estructura de gobierno que se requiere para evaluar y supervisar las transacciones con los proveedores extranjeros (Verwaal y Donkers, 2002). Los costos de transacción podrían ser menores porque estas empresas multinacionales tienen más poder de negociación, lo que limita el comportamiento oportunista de los proveedores extranjeros. Por lo tanto:

Hipótesis 3: La relación entre el outsourcing y las exportaciones es positiva moderada por el tamaño de la empresa.

Las empresas multinacionales podrían ser diferentes en términos de su capacidad de absorción. En nuestro caso, esa capacidad determina su forma de reconocer el valor de los conocimientos y los recursos externos en el extranjero, además de asimilarlo y aplicarlo, posteriormente, en los mercados de exportación. Esta capacidad de absorción está influida por los diversos tipos de conocimientos y recursos que posee la empresa (Eriksson y Chetty, 2003). Argumentándose que la capacidad de absorción puede depender del conocimiento y de los recursos internos que se desarrollan cuando las empresas multinacionales adquieren los productos intermedios en el extranjero.

No sólo a través del outsourcing externo las empresas multinacionales adquieren recursos (servicios o desarrollos), sino que también se apoyan a través de sus sucursales o filiales, lo que les permite absorber conocimientos o know how externos más fácilmente $\mathrm{y}$, por lo tanto, pueden incrementar sus utilidades netas a través del outsourcing. En efecto, existe evidencia empírica en la literatura sobre los be-

${ }^{6}$ La pequeña cantidad de evidencia empírica confirma que las grandes empresas subcontratan más en el extranjero (Mol et al., 2005). 
neficios que obtienen los recursos internos de los externos, debido a su complementariedad (Cassiman y Veugelers, 2006). Cabe mencionar que dicha evidencia también se encuentra en la literatura de administración estratégica (Parmigiani, 2007; Rothaermel, Hitt y Jobe, 2006).

Por otro lado, cuando las empresas organizan el comercio intrafirma, la matriz y sus filiales están más integradas y conectadas. Esto fomenta la comunicación y presentación de informes (Rangan, 2000) dando como resultado el aumento de las ventajas de información de contratación en el extranjero. De igual forma, sostienen que la capacidad de absorción también puede depender de los conocimientos y recursos internos que se han desarrollado junto con la actividad de exportación. La experiencia de la exportación puede aumentar la capacidad de las empresas para buscar, interpretar y utilizar la información de exportación (Cadogan, Diamantopoulos y Siguaw, 2002; y Souchon y Diamantopoulos, 1996); por ejemplo, las empresas con experiencia exportadora pueden prestar menos atención a la nueva información de proveedores extranjeros y otros agentes en los países de importación, a pesar de que esta información podría ser de importancia en los mercados de exportación; además, son menos capaces no sólo de identificar el valor de la información y el conocimiento, sino también para aprovecharla e incorporarla en sus operaciones de exportación existentes.

Aunque el objetivo de este trabajo no es el estudio de los antecedentes de la externalización al extranjero, se identifican dos vías de interacciones entre las exportaciones (de bienes finales) y las importaciones (de bienes intermedios). Se argumenta que las exportaciones antes podrían moderar de manera positiva el impacto del outsourcing externo, lo que rara vez se ha estudiado empíricamente, incluso en la literatura sobre la conexión interior-exterior (Karlsen et al., 2003). Con base en lo anterior, podemos plantear:

Hipótesis 4: Ceteris paribus, los efectos positivos del outsourcing en el desempeño de las exportaciones son mayores cuando las empresas también importan productos intermedios en el grupo multinacional.

Hipótesis 5: La relación entre outsourcing y las exportaciones es moderada positivamente por la experiencia de exportación de la empresa. 


\section{Metodología}

Las fuentes de información son el Directorio de exportadores de Bancomext y las Estadísticas de Comercio Exterior, desarrolladas por el Grupo de Trabajo de Estadísticas de Comercio Exterior, integrado por el Banco de México, el Instituto Nacional de Estadística, Geografía e Informática, el Servicio de Administración Tributaria y la Secretaría de Economía.

Cubriendo alrededor del $70 \%$ de las exportaciones y las importaciones de fabricación mexicana para cada fracción arancelaria, la base de datos describe, en el nivel de producto, la distribución de las importaciones por país de origen y las exportaciones por país de destino. Por cada transacción que las empresas comprometen se indica el valor y el tipo de bienes que se comercializan (bienes intermedios $v s$. bienes finales), así como si la operación se ha establecido con los proveedores independientes (el outsourcing externo) o con filiales en el extranjero (intraempresa de abastecimiento). Ahora bien, el outsourcing se define como los bienes intermedios suministrados por proveedores independientes de extranjeros (Kotabe y Murray, 2004; Lei y Hitt, 1995; Mol et al., 2005.). Debido a la naturaleza transversal de los datos, se analizan los resultados del outsourcing en un punto dado del tiempo y no su proceso; de esta forma no podemos distinguir entre la sustitución basada en favor y una abstención basada en la externalización (Holcomb y Hitt, 2007; Rasheed y Gilley, 2005). Las empresas llevan a cabo la actividad de coordinación interna antes de subcontratar, o se han basado en proveedores externos desde el principio. En las conclusiones se plantearan las limitaciones de nuestros datos.

De las bases de datos mencionadas anteriormente se obtiene una muestra equivalente que incluye alrededor de mil empresas. Al control de los factores determinantes en el nivel país, estamos obligados a reducir el país de destino de las exportaciones de alrededor de 70 mercados de exportación (incluidos los principales países desarrollados y en desarrollo). Para estos países se dispone de datos exhaustivos sobre el PIB (Banco Mundial) y la distancia cultural ${ }^{7}$ con México. Hay que tomar en cuenta el cálculo de la variable del outsourcing (véase la sección siguiente), el cual no se ve afectado por esta restricción. Las empresas compran bienes intermedios de alrededor de 140 países importadores.

${ }^{7}$ http:// www.geert-hofstede.com/ 
Para el establecimiento de las mediciones, tenemos que definir cuáles son las variables dependientes e independientes.

En primer lugar definiremos las variables dependientes. Frecuentemente, en la literatura de administración, se mide el desempeño de las exportaciones por las ventas de exportación de bienes finales (Ito y Pucik, 1993), lo que supone que las empresas crean valor a partir de las exportaciones. Las ventas de exportación (indicado por la variable de exportación) se calculan para cada empresa del país; a su vez, para cada una de ellas se calculan las ventas de exportación en cada uno de los 70 países de exportación de nuestra muestra. No hemos mantenido la intensidad de la exportación (es decir, la proporción de las ventas de exportación en las ventas totales), aunque esta medida proporciona resultados similares. Se podría interpretar erróneamente un cambio en la intensidad de exportación porque el outsourcing puede afectar a la exportación y las ventas nacionales.

Para las variables independientes, se tiene que la variable principal define el outsourcing (véase el Anexo A2). Para calcularla se suman para cada empresa el valor de los insumos suministrados por proveedores independientes extranjeros. Menos del $10 \%$ de las empresas no subcontratan en el extranjero. Esta medida se divide entre las ventas totales en el control de los efectos de tamaño para reducir el nivel de correlación con el tamaño variable, que se mide por el número de empleados; cabe señalar que alrededor de la mitad de las empresas tienen menos de 250 empleados (es decir, son las Pyme). El tamaño de la empresa se utiliza con frecuencia como sustituto de la empresa de recursos (Dhanaraj y Beamish, 2003) o economías de escala. Es importante considerar que nuestros resultados no son sensibles a la medida del outsourcing adoptada en el extranjero.

Para investigar la hipótesis 2 se incluye una variable ficticia (dummy) Comercio Bilateral, que toma el valor 1 cuando los países de importación y exportación son idénticos. Esta variable dummy, que se construye para cada par de países, se puso en interacción con la variable de outsourcing (variable outsourcing $\times$ Comercio Bilateral). Para la hipótesis 3 se incluye la variable interactiva entre outsourcing y tamaño (variable outsourcing x tamaño). Para probar la hipótesis 4 creamos una variable dummy sobre aprovisionamiento dentro de las empresas y su variable interactiva outsourcing $\times$ intrafirma; esta variable toma el valor 1 si la empresa importa insumos dentro de la red sobre las EMN y 0 en caso contrario; cabe mencionar que alrededor del $40 \%$ de las empresas manejan las importaciones intrafirma. Asimismo, dentro de las empresas de abastecimiento deberían mejorar los resultados 
de éstas (Kotabe y Murray, 2004). Por último, para poner a prueba la hipótesis 5, experimentamos medida a la exportación (variable experiencia de la exportación) como el número de años que las empresas han exportado.

Es importante interactuar con la variable de outsourcing (variable de outsourcing $\times$ experiencia en la exportación). Las empresas que están más familiarizadas con los mercados de exportación se prevé que exporten más. Para la hipótesis 3 no se encontró evidencia de efecto invertido en forma de U asociadas con el tamaño variable. Después de un cierto umbral, un aumento en el tamaño de la empresa podría haber afectado la eficiencia de los recursos de la empresa. Para la hipótesis 4, y en relación con el aprovisionamiento, se construyó la variable dummy dentro de la empresa para cada empresa del país, sin observarse cambios importantes.

Variables de control. Las variables de control influyen en las exportaciones; En el nivel de las empresas, además de las variables tamaño, aprovisionamiento dentro de las empresas y la experiencia de exportación, se añade la variable de los costos laborales unitarios (relación costo del trabajo sobre el valor añadido). Ceteris paribus, un mayor costo unitario del trabajo reduce las ventajas basadas en los costos y la capacidad de exportación. Este costo puede variar de forma independiente de las decisiones de contratación externa debido al proceso de innovación o nuevas prácticas de gestión. En el nivel nacional se añade el tamaño del mercado de exportación (es decir, el PIB), la distancia geográfica y cultural (Morosini, Shane y Singh, 1998) entre México y los países de exportación, indicado por las variables del PIB, así como, la distancia geográfica y la distancia cultural, respectivamente. Las empresas esperan exportar más a los mercados que son más grandes y menos distantes — geográficamente o culturalmente. Por último, se introducen las variables dummy fijas del sector para controlar las diferencias observadas entre las industrias.

\section{Modelo econométrico}

En este análisis transversal se toma el logaritmo de las exportaciones porque se ha demostrado en la literatura empírica que se obtiene un mejor ajuste con respecto a los datos del comercio (Stein y Daude, 2007). Sin embargo, la variable de exportación contiene un gran número de ceros. Por lo tanto, calcular su logaritmo excluirá a cero observaciones, que pueden dar lugar a un sesgo de estimación seria. Una solución frecuente es utilizar el registro de la transformación log (Export+1). Log (Export) es aproximadamente igual a log (Export+1) para grandes valores de exportación, como en nuestro caso. Entonces, en nuestro modelo 1 empleamos 
mínimos cuadrados ordinarios (MCO) del modelo con errores estándar robustos; sin embargo, se queda sólo con los flujos positivos de las exportaciones o mediante el procedimiento de Heckman en dos etapas que conducen a una conclusión similar. En el modelo 2 se controla por la endogeneidad y simultaneidad, pues las exportaciones podrían afectar al outsourcing, como se mencionó anteriormente. Usamos las dos etapas mínimos cuadrados (MC2E) enfoque (Salomón y Shaver, 2005; Wooldridge, 2002). Tomamos la tasa del impuesto a las sociedades y los usuarios de Internet - por cada mil habitantes- (Banco Mundial) en los países importadores como instrumentos. Estas variables, Impuestos e Internet, son vistas como indicadores del contexto de los negocios locales y la infraestructura del país. Se utilizan variables en el costo de la mano de obra y el nivel educativo (Banco Mundial) como sustitutos de los costos de producción y el capital humano, lo cual no parece influir en los resultados, aunque reduce la muestra.

Es importante considerar que todas las variables explicativas se refieren al año 1999 - quedando variables de control de 1 año, tales como el tamaño, los costos laborales unitarios o el PIB que no modifican los resultados- y se expresan en logaritmos (con la excepción de las variables dummy) ${ }^{8}$. Estos coeficientes log-log de los modelos de variable pueden ser interpretados simplemente como la elasticidad, que facilitan la comparación entre las variables explicativas.

\section{Resultados}

En relación con la evaluación de los efectos de la deslocalización de las ventas de exportación, en el cuadro 1 se presentan los principales hallazgos?

En el modelo 1 (MCO), las columnas 1-5 investigan las hipótesis 1-5, respectivamente, mientras que las columnas 6-10 replican estas estimaciones para el modelo 2 (MC2E). En los modelos 1 y 2 no se detectó multicolinealidad severa. En todas las estimaciones el valor de la varianza del factor de inflación es menor que el umbral crítico del 10. Por otra parte, se realizó un F-test incremental. Hemos comprobado que la inclusión de las variables interactivas contribuye a un aumento significativo en el ajuste a los datos.

\footnotetext{
${ }^{8}$ Para las variables de tomar los valores cero, se utiliza otra vez el registro de la transformación $(\mathrm{X}+\mathrm{a})$ cuando el valor positivo de una es muy pequeña en relación con X.

${ }^{9}$ Considere que en el cuadro 1 , la variable de comercio bilateral se incluye sólo en las columnas 2 y 7 . La inclusión de esta variable como una variable de control en otras especificaciones no afecta a nuestros resultados.
} 


\section{Cuadro 1 \\ Efectos del outsourcing en los resultados de la exportación}

\begin{tabular}{|c|c|c|c|c|c|c|c|c|c|c|}
\hline \multirow{3}{*}{$\begin{array}{c}\text { CONCEPTOS } \\
\text { Tamaño }\end{array}$} & \multicolumn{2}{|c|}{ Modelo 1 Modelo 1} & Modelo 1 & 1 Modelo 1 & Modelo 1 & Modelo 2 & Modelo 2 & Modelo 2 & Modelo 2 & Modelo 2 \\
\hline & $0.356^{* * *}$ & $0.315^{\star \star *}$ & $0.351^{* * *}$ & $0.358^{* * *}$ & $0.356^{* * *}$ & $0.356^{\star * *}$ & $0.317^{* * *}$ & $0.325^{* * *}$ & $0.353^{\text {t*t }}$ & $0.362^{* * *}$ \\
\hline & $(0.007)$ & $(0.006)$ & $(0.009)$ & $(0.007)$ & $(0.007)$ & $(0.007)$ & $(0.007)$ & $(0.006)$ & $(0.007)$ & $(0.007)$ \\
\hline $\begin{array}{l}\text { Los costos } \\
\text { laborales unitarios }\end{array}$ & $-0.260^{\star \star \star *}$ & $-0.240^{\star \star \star}$ & $-0.259^{* * *}$ & $-0.262^{+\star \star}$ & $-0.261^{\star \star \star}$ & $-0.285^{* \star *}$ & $-0.262^{\star * \star}$ & $-0.271^{\star \star *}$ & $-0.287^{*+*}$ & $-0.274^{\star \star *}$ \\
\hline & $(0.018)$ & $(0.018)$ & $(0.019)$ & $(0.018)$ & $(0.018)$ & $(0.020)$ & $(0.020)$ & $(0.049)$ & $(0.020)$ & $(0.020)$ \\
\hline $\begin{array}{l}\text { La experiencia de } \\
\text { exportación }\end{array}$ & $0.035^{* * *}$ & $0.034^{* * *}$ & $0.044^{* * *}$ & $0.034^{* \star *}$ & $0.046^{* * *}$ & $0.037^{*+* t}$ & $0.036^{* * *}$ & $0.050^{*+*}$ & $0.036^{* \star *}$ & $0.051^{* * *}$ \\
\hline & $(0.001)$ & $(0.001)$ & $(0.002)$ & $(0.001)$ & $(0.002)$ & $(0.001)$ & $(0.001)$ & $(0.002)$ & $(0.001)$ & $(0.008)$ \\
\hline PIB & $0.311^{* * *}$ & $0.253^{\text {t+*t }}$ & $0.311^{* * *}$ & $0.312^{*+*}$ & $0.311^{* * *}$ & $0.325^{*+* t}$ & $0.267^{+* *}$ & $0.326^{* * *}$ & $0.325^{*+*}$ & $0.326^{* * *}$ \\
\hline & $(0.004)$ & $(0.003)$ & $(0.004)$ & $(0.004)$ & $(0.005)$ & $(0.004)$ & $(0.004)$ & $(0.004)$ & $(0.005)$ & $(0.004)$ \\
\hline $\begin{array}{l}\text { Distancia } \\
\text { Geográfica }\end{array}$ & $-0.400^{\star \star \star}$ & $-0.316^{\star \star *}$ & $-0.400^{* \star *}$ & $-0.399^{\star \star \star}$ & $-0.400^{\star \star \star \star}$ & $-0.424^{\star \star \star}$ & $-0.337^{\star \star \star}$ & $-0.423^{\star \star \star}$ & $-0.423^{\star \star *}$ & $-0.424^{* * *}$ \\
\hline & $(0.007)$ & $(0.007)$ & $(0.007)$ & $(0.008)$ & $(0.007)$ & $(0.008)$ & $(0.008)$ & $(0.007)$ & $(0.008)$ & $(0.007)$ \\
\hline Distancia Cultural & $-0.446^{*+*}$ & $-0.414^{* * *}$ & $-0.445^{*+*}$ & $-0.445^{* * *}$ & $-0.447^{+*+*}$ & $-0.460^{*+*}$ & $-0.425^{*+*+}$ & $-0.460^{* * *}$ & $-0.458^{*+*}$ & $-0.461^{*+* t}$ \\
\hline & $(0.021)$ & $(0.021)$ & $(0.022)$ & $(0.020)$ & $(0.021)$ & $(0.023)$ & $(0.023)$ & $(0.022)$ & $(0.022)$ & $(0.023)$ \\
\hline $\begin{array}{l}\text { Outsourcing } \\
\text { interno }\end{array}$ & $0.068^{* * *}$ & $0.031^{* *+*}$ & $0.041^{*}$ & $0.050^{* *}$ & $0.049^{\star \star \star}$ & $0.073^{\star \star \star}$ & $0.066^{\star \star \star}$ & $0.062^{* *}$ & $0.065^{* *}$ & $0.071^{* * *}$ \\
\hline & $(0.012)$ & $(0.012)$ & $(0.024)$ & $(0.020)$ & $(0.016)$ & $(0.028)$ & $(0.025)$ & $(0.031)$ & $(0.033)$ & $(0.024)$ \\
\hline Outsourcing & $0.073^{*+*}$ & $0.041^{* * t}$ & $0.121^{* * *}$ & $0.051^{* * *}$ & $0.053^{* * *}$ & $0.358^{* * *}$ & $0.320^{* * *}$ & $0.509^{* * *}$ & $0.381^{* *+}$ & $0.397^{\star \star \star}$ \\
\hline & $(0.005)$ & $(0.004)$ & $(0.005)$ & $(0.006)$ & $(0.004)$ & $(0.017)$ & $(0.017)$ & $(0.026)$ & $(0.025)$ & $(0.019)$ \\
\hline Comercio Bilateral & & $2.239^{*+*}$ & & & & & $1.431^{* * *}$ & & & \\
\hline & & $(0.113)$ & & & & & $(0.126)$ & & & \\
\hline $\begin{array}{c}\text { Outsourcing } \times \\
\text { Comercio Bilateral }\end{array}$ & & $0.168^{*+* t}$ & & & & & $0.118^{* *}$ & & & \\
\hline & & $(0.044)$ & & & & & $(0.050)$ & & & \\
\hline $\begin{array}{c}\text { Outsourcing } \times \\
\text { Tamaño }\end{array}$ & & & $0.027^{* * t}$ & & & & & $0.169^{* * \star}$ & & \\
\hline & & & $(0.002)$ & & & & & $(0.020)$ & & \\
\hline $\begin{array}{c}\text { Outsourcing } \\
\times \text { Outsourcing } \\
\text { intrafirma }\end{array}$ & & & & $0.089^{+* * *}$ & & & & & $0.294^{* * t}$ & \\
\hline & & & & $(0.004)$ & & & & & $(0.027)$ & \\
\hline $\begin{array}{l}\text { Outsourcing } \times \\
\text { experiencia de la } \\
\text { exportación }\end{array}$ & & & & & $0.015^{*+*}$ & & & & & $0.094^{* * *}$ \\
\hline & & & & & $(0.001)$ & & & & & $(0.003)$ \\
\hline Constante & $-3.723^{*+* t}$ & $-3.210^{\star \star * *}$ & $-3.717^{* * *}$ & $-3.741^{* * *}$ & $-3.737^{\star \star \star}$ & $-5.861^{\star * * t}$ & $-5.215^{+* *}$ & $-5.325^{* *}$ & $-5.660^{* * *}$ & $-5.754^{\star \star \star}$ \\
\hline & $(0.098)$ & $(0.095)$ & $(0.103)$ & $(0.098)$ & $(0.098)$ & $(0.243)$ & $(0.248)$ & $(0.314)$ & $(0.245)$ & $(0.244)$ \\
\hline Observaciones & 146,365 & 146,365 & 146,365 & 146,365 & 146,365 & 135,234 & 135,234 & 135,234 & 135,234 & 135,234 \\
\hline R 2 & 0.15 & 0.17 & 0.16 & 0.16 & 0.16 & 0.13 & 0.15 & 0.14 & 0.14 & 0.14 \\
\hline
\end{tabular}

Notas:

Errores estándar robustos entre paréntesis.

Sector efectos fijos están incluidos. Todas las variables explicativas (con la excepción de las variables dummy) se expresan en logaritmos.

Dependientes y las variables explicativas se refieren al año 1999.

Modelo 1, MCO; Modelo 2, MC2E.

* Significativo al $10 \%$, ** significativo al $5 \%$, *** significativo al $1 \%$. 
En los modelos 1 y 2 de la variable de outsourcing es positiva y significativamente diferente de cero al 1\%, en apoyo a nuestra primera hipótesis. El tamaño del coeficiente (aquí interpretado como una elasticidad) varía a través de nuestras diferentes estimaciones de 0.041 a $0.509 .{ }^{10} \mathrm{Si}$ bien parece ser pequeña en el modelo 1 , el tamaño del efecto es mucho mayor en el modelo 2 cuando se controla por la simultaneidad potencial y endogeneidad.

En el modelo 2, el efecto de la externalización variable es más importante que la de la otra empresa en el nivel tamaño variable o en los costos laborales unitarios. Ceteris paribus, el outsourcing en el extranjero ayuda a las empresas a exportar más a través de ahorro de costos, mayor flexibilidad, los recursos adicionales y/o conocimiento del mercado.

En cuanto a las hipótesis 2-5, éstas también se apoyan empíricamente en ambos modelos ( 1 y 2 ). La variable Comercio Bilateral y su variable interactiva Outsourcing $\times$ Comercio Bilateral (hipótesis 2), así como el tamaño de las variables y Tamaño de outsourcing $\times$ (hipótesis 3 ), dentro de las empresas de outsourcing $\times$ Abastecimiento intrafirma (hipótesis 4), experiencia de la exportación y outsourcing $\times$ experiencia de exportación (hipótesis 5), muestran un signo positivo y significativo.

No hay que olvidar, finalmente, que las variables de control tienen los signos previstos y son importantes, lo que subraya el buen ajuste general de nuestro modelo empírico. ${ }^{11}$ En el nivel de las empresas el costo laboral unitario muestra un signo negativo, mientras que el tamaño de la empresa, la experiencia de la exportación o la organización de las importaciones dentro de las empresas tienden a aumentar las exportaciones. En el plano nacional, los flujos de la distancia geográfica y cultural impiden la exportación; por otro lado, los mercados más grandes estimulan las exportaciones.

\footnotetext{
${ }^{10}$ Un coeficiente de 0.509 se interpreta de la siguiente manera: la variable aumenta la exportación de $0.509 \%$ cuando la variable de outsourcing aumenta en un $1 \%$.

${ }^{11}$ En el cuadro 1 el valor de R2 es relativamente bajo; sin embargo, una R2 baja se observa típicamente en datos de corte transversal con un gran número de observaciones [véase Gujarati (2004), por un breve debate, o, por ejemplo, Wooldridge (2009)].
} 


\section{Conclusiones}

En este trabajo se examina el impacto del outsourcing en el desempeño de las exportaciones de las empresas. Se hace una contribución empírica a la literatura de administración, pues no existen estudios microeconómicos en el outsourcing externo (Salomón y Shaver, 2005). Aunque el artículo se llevó a cabo de forma independiente, complementa el artículo recientemente publicado por Di Gregorio et al. (2009), quienes sugieren - pero no lo prueban — que el outsourcing no podía ser beneficioso para las grandes empresas multinacionales.

En este artículo se argumenta y demuestra que, en promedio, la externalización al extranjero aumenta la competitividad internacional de las empresas multinacionales. Sin embargo, analizando a profundidad los beneficios y los costos de la externalización al extranjero, se explica que los efectos del outsourcing internacional pueden estar condicionados a las características de exportación de cada país y empresa determinada; esta hipótesis puede ser muy probable que también se verifique para las Pyme.

Los resultados empíricos apoyan esta hipótesis, destacando algunas condiciones en las que las ganancias de exportación podrían ser mayores (o menores). Mostramos que los efectos positivos asociados con la externalización al extranjero son más directos y, por lo tanto, más fuertes dentro del mercado de exportación para la empresa de subcontrata. La importación de insumos intermedios en el mercado de exportación da a las empresas información sobre los mercados locales, lo que reduce las desventajas específicas de cada lugar y, así, fortalece su presencia en el mercado de exportación.

Por otra parte, el impacto de la externalización al extranjero es probable que sea heterogéneo entre las empresas multinacionales. Esta heterogeneidad refleja diferencias en los recursos y las capacidades de absorción entre las empresas multinacionales, características que son esenciales para el éxito de la externalización al extranjero. La moderación de los costos de transacción y coordinación, que van unidas con el outsourcing, determinan la capacidad de la empresa para asimilar y explotar los recursos externos y el conocimiento en los mercados de exportación. En concordancia, por ejemplo, con trabajos empíricos sobre la multinacionalidad y el rendimiento (Bausch \& Krist, 2007) encontramos que la gran mayoría puede ayudar a las empre- 
sas a través de efectos de escala y recursos para superar las dificultades estratégicas y operativas que surgen con el outsourcing externo. Asimismo, esos trabajos señalan el conocimiento y los recursos que se desarrollan cuando las empresas de origen extranjero dentro de la red sobre las EMN — o de exportación - tienen efectos positivos de moderación en el desempeño de la estrategia del outsourcing.

Este trabajo ofrece implicaciones para el programa de investigación en el outsourcing externo a futuro. En primer lugar, hay una necesidad de explorar aún más la heterogeneidad en el desempeño de la empresa como se ha hecho, por ejemplo, en el campo de la investigación relativa a las fusiones y adquisiciones en la estrategia o en la multinacionalidad en el desempeño de las empresas en los negocios internacionales. El conjunto de factores internos y externos que pueden moderar el impacto del outsourcing siguen estudiándose en la literatura estratégica y de negocios internacionales. Por otra parte, todavía tenemos evidencia microeconómica muy limitada en la conexión del interior hacia el exterior en los mercados mundiales (Karlsen et al., 2003). Este trabajo contribuye a llenar esta laguna empírica, no sólo en explorar el impacto de las importaciones (es decir, el outsourcing externo) en las exportaciones, sino también consideran lo contrario, aunque está fuera de nuestro alcance para examinar los antecedentes de la externalización al extranjero; por ello, se toma en cuenta, teórica y empíricamente, que las exportaciones pueden influir en las importaciones. En general, se requiere más investigación sobre la estrategia integrada que las empresas pueden desarrollar en los mercados mundiales, sobre cómo las operaciones de perfeccionamiento activo-pasivo se articulan, y si los vínculos de dos vías pueden crear sinergias a través de fronteras y operaciones.

La estrategia global que algunas empresas multinacionales han llevado a cabo a través de una exclusiva red de afiliados y dentro de las empresas de abastecimiento ha sido relativamente bien documentada por los investigadores académicos y practicantes empresariales.

Con la liberalización del comercio y el progreso tecnológico, las empresas multinacionales, sin embargo, se alejaron de la integración vertical internacional moviéndose hacia el outsourcing de forma progresiva mediante una red global de proveedores independientes. Esta nueva estructura organizativa, que combina ambas empresas subsidiarias y proveedores (es decir, dentro de las empresas de abastecimiento y el outsourcing externo) en los distintos países, ha sido mucho menos estudiada, por lo que se hace un llamado para obtener más evidencia em- 
pírica sistemática sobre el abastecimiento global y su papel en la estrategia de las empresas multinacionales, así como para el desarrollo de un amplio marco teórico (Ghemawat, 2007). ${ }^{12}$

Los resultados también tienen implicaciones tanto para políticos como profesionales. En este reciente periodo de turbulencia económica y financiera, los gobiernos podrían verse tentados a volver al proteccionismo. Estos resultados sugieren que esta decisión política podría ser costosa porque no sólo supone un perjuicio para el comercio internacional de bienes finales, sino también la de bienes intermedios. En los mercados globales, donde las empresas nacionales tienden cada vez más a subcontratar a los proveedores extranjeros, el outsourcing puede ser un factor determinante de la competitividad internacional. Las medidas proteccionistas en contra de la externalización al extranjero podrían ser contraproducentes debido a que perjudicaría a la cuantía de las exportaciones de los países.

Los resultados también sugieren el papel estratégico del outsourcing en el aumento global de las ventajas competitivas, en las ventajas de costos y en la externalización al extranjero en el mercado de recursos internacionales, pues es una manera de obtener recursos externos altamente complementarios, así como para el desarrollo del conocimiento. En los mercados en rápido movimiento, en particular, una sola empresa no puede mantener todos los recursos que se requieren para crecer y prosperar; sin embargo, para beneficiarse plenamente del outsourcing, las empresas deben facilitar la transferencia de conocimientos dentro de la organización entre sus diversas funciones y departamentos: entre la oferta y la actividad de exportación dentro de cada afiliado, así como entre las diferentes actividades de suministro dentro de la red EMN. Por otra parte, el compartir los conocimientos internos podría aumentar no sólo la transferencia internacional de recursos de las empresas multinacionales, sino también, su capacidad de absorción, la cual podría aumentar, a su vez, la transferencia de conocimientos entre organizaciones.

Finalmente, se discuten las diversas limitaciones de nuestra base de datos que ofrecen posibilidades para nuevas investigaciones. En primer lugar, la base de datos no

\footnotetext{
${ }^{12}$ Recientemente, revisando el concepto de estrategia global en un nuevo marco denominado triángulo AAA (adaptación de la agregación, el arbitraje) Ghemawat (2007) sostiene que, para crear valor, las empresas tienen que pensar en cómo explotar - y no sólo la manera de adaptar y superar- las diferencias entre países a través de la especialización internacional vertical (es decir, el outsourcing y/o de abastecimiento dentro de las empresas).
} 
informa de la identidad de los proveedores extranjeros, por lo que no nos permiten explorar los efectos de interacción entre compradores y proveedores (por ejemplo, la complementariedad de recursos); del mismo modo, no podemos examinar la heterogeneidad entre países en el desempeño de las empresas de outsourcing. Así, este estudio, el cual se avoca al caso mexicano, debe ser replicado para los diferentes contextos económicos y los mercados de recursos. En segundo lugar, la base de datos se llevó a cabo para el año 2007; sería interesante explorar los efectos de la externalización al extranjero varios años después.

Los avances en tecnología de la información y la comunicación, así como la experiencia de externalización, pueden aumentar los efectos positivos del outsourcing; de igual forma, algunos efectos negativos pueden aparecer a largo plazo solamente (por ejemplo, el riesgo de dependencia y la pérdida de competencias). Los datos de panel también podrían ayudar a comprender la distinción entre alta mar y una abstención — la sustitución basada en el outsourcing y sus implicaciones. En el caso de la abstención basada en el outsourcing, la capacidad de absorción de las empresas de contratación externa podría ser más débil porque no han desarrollado una experiencia interna y los conocimientos específicos de la actividad que se externaliza; por otra parte, dado que la actividad no se ha hecho antes, la abstención basada en la externalización al extranjero es menos probable que afecte la actual organización de la empresa (las rutinas organizacionales existentes) o afecte, por ejemplo, la motivación de los empleados (Puranam y Srikanth, 2007) y a los gastos de interrupción, en el caso de las fusiones y adquisiciones.

\section{Referencias}

Anand, J. y A. Delios (1997). Location specificity and the transferability of downstream assets to foreign subsidiaries. Journal of International Business Studies 28 (3): 579-603.

Barkema, H., J. Bell y J. Pennings (1996). Foreign entry, cultural barriers and learning. Strategic Management Journal 17 (2): 151-166.

Bausch, A. y M. Krist (2007). The effect of context-related moderators on the internationalization-performance relationship: evidence from meta-analysis. Management International Review 47 (3): 319-347. 
Cadogan, J., A. Diamantopoulos y J. Siguaw (2002). Export market-oriented activities: their antecedents and performance consequences. Journal of International Business Studies 33 (3): 615-626.

Cassiman, B. y R. Veugelers (2006). In search of complementarity in innovation strategy: internal R\&D and external knowledge acquisition. Management Science 52 (1): 68-82.

Choi, C., S. Lee y J. Kim (1999). A note on countertrade: contractual uncertainty and transaction governance in emerging economies. Journal of International Business Studies 30 (1): 189-201.

Dhanaraj, C. y P. Beamish (2003). A resource-based approach to the study of export performance. Journal of Small Business Management 41 (3): 242-261.

Di Gregorio, D., M. Musteen y D. Thomas (2009). Offshore outsourcing as a source of international competitiveness for SMEs. Journal of International Business Studies 40 (6): 969-988.

Doh, J., K. Bunyaratavej y E. Hahn (2009). Separable but not equal: the location determinants of discrete services off shoring activities. Journal of International Business Studies 40 (6): 926-943.

Elmaghraby, W. (2000). Supply contract competition and sourcing policies. Manufacturing and Service Operations Management 2 (4): 350-371.

Eriksson, K. y S. Chetty (2003). The effect of experience and absorptive capacity on foreign market knowledge. International Business Review 12 (6): 673-695.

Farrell, D. (2005). Off shoring: value creation through economic change. Journal of Management Studies 42 (3): 675-683.

Friedman, T. (2004). What goes around? New York Times 26 February: 27.

Ghemawat, P. (2007). Managing difference: the central challenge of global strategy. Harvard Business Review 85 (3): 58-68. 
Grant, R. (1991). The resource-based theory of competitive advantage: implications for strategy formulation. California Management Review 33 (3): 114-135.

Gujarati, D. N. (2004). Basic econometrics. New York: McGraw-Hill.

Holcomb, T. y M. Hitt (2007). Toward a model of strategic outsourcing. Journal of Operations Management 25 (2): 464-481.

Ito, K. y V. Pucik (1993). R\&D spending, domestic competition and export performance of Japanese manufacturing firms. Strategic Management Journal 14 (1): 61-75.

Karlsen, T., P. Silseth, G. Benito y L. Welch (2003). Knowledge, internationalization of the firm, and inward-outward connections. Industrial Marketing Management 32 (5): 385-396.

Kotabe, M. y R. Mudambi (2009). Global sourcing and value creation: opportunities and challenges. Journal of International Management 15 (2): 121-242.

Kotabe, M. y J. Murray (2004). Global sourcing strategy and sustainable competitive advantage. Industrial Marketing Management 33 (1): 7-14.

Lei, D. y M. Hitt (1995). Strategic restructuring and outsourcing: the effect of mergers and acquisitions and LBOs on building firm skills and capabilities. Journal of Management 21 (5): 835-859.

Leiblein, M., J. Reuer y F. Dalsace (2002). Do make or buy decisions matter? The influence of organizational governance on technological performance. Strategic Management Journal 23 (9): 817-833.

Levy, D. (2005). Off shoring in the new global political economy. Journal of Management Studies 42 (3): 685-693.

Mesquita, L., J. Anand y T. Brush. (2008). Comparing the resource-based and relational views: knowledge transfer and spillover in vertical alliances. Strategic Management Journal 29 (9): 913-941. 
Mol, M., R. Van Tulder y P. Beije (2005). Antecedents and performance consequences of international outsourcing. International Business Review 14 (5): 599-617.

Morosini, P., S. Shane, y H. Singh (1998). National cultural distance and crossborder acquisition performance. Journal of International Business Studies 29 (1): 137-158.

Navaretti, G. y J. Venables (2004). Multinational firms in the world economy. NJ: Princeton University Press.

Parmigiani, A. (2007). Why do firms both make and buy? An investigation of concurrent sourcing. Strategic Management Journal 28 (3): 285-311.

Puranam, P. y K. Srikanth. (2007). What they know vs. what they do: how acquirers leverage technology acquisitions. Strategic Management Journal 28 (8): 805-825.

Rangan, S. (2000). Search and deliberation in international exchange: microfoundations to some macro patterns. Journal of International Business Studies 31 (2): 205-222.

Rasheed, A. y M. Gilley (2005). Outsourcing: national- and firm-level implications. Thunderbird International Business Review 47 (5): 513-528.

Rothaermel, F., M. Hitt y L. Jobe (2006). Balancing vertical integration and strategic outsourcing: effects on product portfolio, product success and firm performance. Strategic Management Journal 27 (11): 1033-1056.

Salomon, R. y M. Shaver (2005). Export and domestic sales: their interrelationship and determinants. Strategic Management Journal 26 (9): 855-871.

y B. Jin (2008). Does knowledge spill to leaders or laggards? Exploring industry heterogeneity in learning by exporting. Journal of International Business Studies, 39 (1): 132-150. 
Souchon, A. y A. Diamantopoulos (1996). A conceptual framework of export market information use: key issues and research propositions. Journal of International Marketing 4 (3): 49-71.

Stein, E. y C. Daude (2007). Longitude matters: time zones and the location of foreign direct investment. Journal of International Economics 71 (1): 96-112.

Verwaal, E. y B. Donkers (2002). Firm size and export intensity: solving an empirical puzzle. Journal of International Business Studies 33 (3): 603-613.

Welch, L. y R. Luostarinen (1993). Inward-outward connections in internationalization. Journal of International Marketing 1 (1): 44-56.

Wooldridge, J. (2002). Econometric analysis of cross section and panel data. Cambridge, MA: MIT Press.

Wooldridge, J. (2009). Introductory econometrics: a modern approach. Mason, $\mathrm{OH}$ : South Western.

Zahra, S. y G. George (2002). Absorptive capacity: a review, reconceptualization and extension. Academy of Management Review 27 (2): 185-203. 


\section{Anexos}

\section{Cuadro A1}

Estadística: las variables en el nivel de empresa

\begin{tabular}{|c|c|c|c|c|c|c|c|}
\hline Variable & Media & d. e. & 1 & 2 & 3 & 4 & 5 \\
\hline 1. Tamaño & 459.8307 & 1140.786 & 1.0000 & & & & \\
\hline $\begin{array}{l}\text { 2. Los costos } \\
\text { laborales } \\
\text { unitarios }\end{array}$ & 0.6832137 & 0.2108186 & -0.0128 & 1.0000 & & & \\
\hline $\begin{array}{l}\text { 3. La experiencia } \\
\text { de } \\
\text { exportación }\end{array}$ & 4.990239 & 2.183602 & 0.1475 & 0.0163 & 1.0000 & & \\
\hline 4. Outsourcing & 0.1117467 & 0.1146129 & 0.0633 & -0.0152 & 0.0309 & 1.0000 & \\
\hline $\begin{array}{l}\text { 5. Outsourcing } \\
\text { dentro de las } \\
\text { empresas }\end{array}$ & 0.4143485 & 0.4926108 & 0.0591 & -0.0107 & 0.0542 & 0.0268 & 1.0000 \\
\hline
\end{tabular}

Resultados de la investigación.

\section{Cuadro A2}

\section{Estadística: Las variables en el nivel país}

\begin{tabular}{|c|c|c|c|c|c|c|c|c|}
\hline Variable & Media & d. e. & 1 & 2 & 3 & 4 & 5 & 6 \\
\hline $\begin{array}{c}\text { 1. Distancia } \\
\text { geográfica }\end{array}$ & 5655.493 & 4177.93 & 1.0000 & & & & & \\
\hline 2. PIB & $2.37 \times 10^{12}$ & $7.43 \times 10^{12}$ & 0.0041 & 1.0000 & & & & \\
\hline $\begin{array}{c}\text { 3. Distancia } \\
\text { cultural }\end{array}$ & 2.505933 & 0.7844219 & 0.2563 & 0.0414 & 1.0000 & & & \\
\hline $\begin{array}{c}\text { 4. Comercio } \\
\text { Bilateral }\end{array}$ & 0.0543533 & 0.2267142 & -0.1885 & 0.2183 & -0.0817 & 1.0000 & & \\
\hline 5. Impuestos & 0.323803 & 0.063713 & -0.2334 & 0.0608 & -0.2060 & 0.0316 & 1.0000 & \\
\hline 6. Internet & 96.87533 & 113.4073 & -0.1164 & 0.2981 & 0.1584 & 0.1459 & -0.0951 & 1.0000 \\
\hline
\end{tabular}

PIB expresado en pesos mexicanos

Fuentes: Banco Mundial, Hofstede, Directorio de Exportadores de Bancomext y las Estadísticas de Comercio Exterior desarrolladas por el Grupo de Trabajo de Estadísticas de Comercio Exterior. 